\title{
Optimization Research on Multi-objects Aircraft Maintenance Shop Scheduling Problem
}

\author{
Shaohua Yang, Ying Wang and Gang Liu* \\ Equipment Management \& Safety Engineering College, Air Force Engineering University, Xi’an Shaanxi, 710051, China \\ ${ }^{*}$ Corresponding author
}

\begin{abstract}
To make the maintenance adapt for the modern aircraft, the modeling and scheduling algorithm of aircraft maintenance shop scheduling problem are discussed. At the base of maintenance shop scheduling modeling referring to the formalization of flexible job shop scheduling problem, genetic algorithm is adopted as framework, where the execution steps are designed, and the coupling operator is introduced to adapt the operation sequence part of chromosome to avoid the situation that the chromosome might violate the coupling constraint and could not decode. Subsequently, applicability and quality of multi-objects scheduling algorithm are verified through maintenance shop example.
\end{abstract}

Keywords-military aircraft; maintenance shop; coupling constraint; multi-objects

\section{INTRODUCTION}

As the reference of aircraft maintenance, maintenance shop scheduling should meet the demands to optimize and integrate task element and resource element. However, the contradiction among scheduling objects and resource elements made maintenance use to mainly rely on the artificial scheduling and coordination, which is inefficient, inflexible and difficult to spread. As a result, discuss the automatic generation of scheduling scheme to made maintenance shop adapt for modern aircraft, will have important theoretical value and practical significance. In the optimization and integration of task element and resource element, process constraint is always the basis of element relationship,so flexible job shop scheduling optimization research could provide help for the aircraft maintenance shop scheduling based on process constraint.

Based on the fitness assignment strategy of Pareto dominance, NSGA ${ }^{[1]}$, NPGA adopted fitness sharing mechanism and niche technology to keep the population diversity and to avoid converging to a single Pareto curve, SPEA $^{[2]}$, SPEA $^{[3]}$, PAES $^{[4]}$, NSGA-II $^{[5]}$, NPGA $^{[6]}$ introduced external population to hold non-dominant vector and derived out the interactive mode and maintenance technology of external population and internal population.

At the same time of achievements for reference, we also need to notice that the process constraint of flexible job shop scheduling problem adopts chain constraint, which is only suit to describe the relationship among the operations in single-component systems. Nevertheless, as the representative of multi-component systems, aircraft maintenance is not only involve the chain constraint of operations in the same task, but also refer to the coupling constraint of operations in the different task. Therefore, we take advantage of scheduling optimization algorithm achievement, expend the existence form of process constraint, and composite the chain constraint and coupling constraint to explore the aircraft maintenance shop scheduling optimization.

\section{Multi-OBJECts AircRAft MaintenAnCE SHOP SCHEDULING MODEL CONSTRUCTION}

The paper chooses formalization of flexible job shop scheduling problem to create model. Let $S_{v}$ be the start time, $p_{v, \mu_{v}}$ be the process time, $e_{v}$ be the finish time of operation $V$.

\section{A. Task Model}

Given a set $J=\left\{J_{i} \mid i=1,2, \ldots, n\right\}$ for tasks, which is composed of the operation set $O=\left\{O_{i j} \mid i=1,2, \ldots, n, j=1,2, \ldots, n_{i}\right\}, n_{i}=\left|J_{i}\right|$ will be the number of operations belonging to $J_{i}$. Task model describes the constraints from the perspective of process constraint:

1. Task attribution constraints: For an arbitrary operation $v \in O$, there exists unique $\rho_{v}=J_{i} \in J$, which denotes the operation $v$ is the component of task $J_{i}$.

2. Chain constraint: For arbitrary operations $v, u$ that belong to the same task, where exists process order. Set $v$ is priori to $u, s_{u} \geq s_{v}+p_{v, \mu_{v}}$ should be meet, and let $J P[v]$ and $J S[v]$ be the chain adjacent predecessor and chain adjacent successor of operation $v$.

3. Coupling constraint: For arbitrary operations $v, u$ that belong to the different task, where exists process order. Set $v$ is priori to $u$, and let $O P[v]$ and $O S[v]$ be the coupling adjacent predecessor and coupling adjacent successor of operation $V$. 


\section{B. Scheduling Model}

Given a set $M=\left\{M_{k} \mid k=1,2, \ldots, m\right\}$ for units, which is composed of the allocation and sequence set $\prod_{k=1}^{m} Q_{k}=\left\{v \mid \mu_{v}=M_{k}\right\}, \quad m_{k}=\left|Q_{k}\right|$ will be the number of operations belonging to $M_{k}$. Scheduling model describes the constraints from the perspective of unit constraint:

1. Unit attribution constraints: For an arbitrary operation $v \in O$, there exists unique $\mu_{v}=M_{k} \in M$, which denotes the operation ${ }^{v}$ will be processed on unit $M_{k}$, and $M_{k}$ could be arbitrarily selected in units set $C_{v}$.

2. Unit constraint: For arbitrary operations $v, u$ that belong to the same unit, where exists process order. Set $v$ is priori to $u, s_{u} \geq s_{v}+p_{v, \mu_{v}}$ should be meet, and let $M P[v]$ and $M S[v]$ be the unit adjacent predecessor and unit adjacent successor of operation $v$.

\section{Object Model}

According to practical demands of maintenance, the paper sets objects in common use as follows:

1. Complete time setting: Let $C_{J_{i}}$ be the complete time of $J_{i}$, and set

$$
f_{1}=\min \max _{J_{i} \in J} C_{J_{i}}
$$

2. Load time setting: Let $L_{k}=\sum_{v \in O} p_{v, M_{k}}$ be the load time of $M_{k}$, and set

$$
\begin{aligned}
& f_{2}=\min \max _{M_{k} \in M} L_{k} \\
& f_{3}=\min \sum_{M_{k} \in M} L_{k}
\end{aligned}
$$

\section{Multi-OBJECTS SchEdUling OptimizATION ALGORITHM DESIGN}

The paper adopts genetic algorithm as framework and introduces the adaptive mechanism of coupling constraint to design the scheduling algorithm around the step.

\section{A. Chromosome Encoding Mechanism Design}

Maintenance shop scheduling needs to consider the operation allocation and operation sequence at the same time, chromosome encoding mechanism could be designed segmentally.
1. The operation allocation part of chromosome is composed of $\sum_{i=1}^{n}\left|J_{i}\right|$ genes, which is sequenced by the priority of $O_{i j}$ belonging to $J_{i}$. For an arbitrary gene $v$, the operation that $v$ denotes is invariant, whose assignment scope is units set $C_{v}$.

2. The operation sequence part of chromosome is composed of $\sum_{i=1}^{n}\left|J_{i}\right|$ genes as well. For an arbitrary gene $v=i$, if the occurrence number of $i$ equals to $j$ by the end of $v$, that means $v$ denotes operation $O_{i j}$.

\section{B. Scheduling Scheme Initialization}

Let $J A$ be the task allocation array, $J A(i)$ denotes the number of allocated operations belonging to $J_{i}, J A(i)=0$; let $P M$ be the unit allocation array, $P M(k)$ denotes the cumulative time of allocated operations belonging to $M_{k}$, $P M(k)=0$; let $A L$ denotes the list that operations to be allocated, $A L=\varnothing$.

1. Test the first operation $O_{i j}$ to be allocated in $J_{i}$ in proper order, and let $O_{s t}$ be the coupling adjacent predecessor of $O_{i j}$. If $\operatorname{JobA}(s) \geq t$, it means that $O_{i j}$ could be allocated, and adds $i$ to the tail of $A L$; otherwise, it means that $O_{i j}$ is not meet the allocation condition.

2. Randomly select an operation in $A L$, and let $O_{i j}$ be the selection, $J A(i)+1$, and wipe $A L$.

3. Randomly select a unit in unit set of $O_{i j}$, and let $M_{k}$ be the selection, $P M(k)+p_{O_{i j}, M_{k}}$.

4. According to chromosome encoding mechanism, set the selection $O_{i j}$ and $M_{k}$ into corresponding genes, and loop execute the step 1 to 4 .

\section{Crossover Operator Design}

Crossover operator simulates the inheritance patterns of biological intergeneration, which inherits the excellent characteristics from parent chromosomes, and needs to be designed into the operation allocation part and the operation sequence part.

1. In operation allocation part, the operation that arbitrary gene $v$ denotes is invariant, the paper adopts uniform cross to design crossover operator.

2. In operation sequence part, the operation that arbitrary gene $v$ denotes is variant, the paper adopts POX cross to design crossover operator. 


\section{Mutation Operator Design}

Mutation operator simulates the mutation patterns of biological intergeneration, which improves population diversity and escapes local minima with a chance, and needs to be designed into the operation allocation part and the operation sequence part.

1. In operation allocation part, the operation that arbitrary gene $v$ denotes is invariant, so that mutation operator could be designed based on the same genes.

(1) Randomly select $N$ genes in operation allocation part of chromosome, $N \in\left[0, \sum_{i=1}^{n}\left|J_{i}\right|\right]$.

(2) Mutate the selected $N$ genes in proper order, and let gene $v$ denotes operation $O_{i j}$, make $v$ randomly assign in the scope of $C_{v}$.

2. In operation sequence part, the operation that arbitrary gene $v$ denotes is variant, so that mutation operator could be designed based on the different genes.

(1) Randomly select $N$ genes in operation sequence part of chromosome, $N \in\left[0, \sum_{i=1}^{n}\left|J_{i}\right|\right]$.

(2) Mutate the selected $N$ genes in proper order, and let gene $V$ select mutation pattern from insert pattern and exchange pattern, where the position is also random.

\section{E. Coupling Operator Design}

The operation sequence part of encoding mechanism that paper designed could only satisfy the demand of chain constraint, so that the chromosome might violate the coupling constraint and could not decode after crossover and mutation. Therefore, the paper introduces the adaptive mechanism of coupling constraint what would be described as coupling operator.

Let $S T$ be the task sequence array, $S T(i)$ denotes the number of operations adapted, $S T(i)=0$; let $S L$ be the list that operations to be adapted, $S L=\varnothing$.

1. Test the first gene $v$ to be adapted in parent chromosome $P(T)$, and let $O_{\text {st }}$ be the coupling adjacent predecessor of $O_{i j}$ what $v$ denotes. If $S T(s) \geq t$, it means that $O_{i j}$ could be adapted, and adds $v$ to the tail of child chromosome $C(T)$, $S T(i)+1$; otherwise, adds $v$ to the tail of list $S L$ that operations to be sequenced.

2. Test the genes in the list $S L$ in proper order, if operation $O_{i j}$ what $v$ denotes satisfy the adaptive condition, move $v$ from $S L$ to the tail of child chromosome $C(T), S T(i)+1$.

3. Loop execute the step 1 to 2 , until all the genes in $P(T)$ are adapted and $S L$ is empty.
Coupling operator reflect the constraint against chromosome sequence, which could be the connection between chain constraint and coupling constraint.

\section{F. Chromosome Decoding Mechanism Design}

Chromosome decoding mechanism is the inverse operation of encoding mechanism, whih should be designed to exclude the semi-activity schedule.

Let $A M$ be the allocation unit array, where $A M(i, j)$ denotes the unit that $O_{i j}$ selected; let $A P$ be the allocation time array, where $A P(i, j)$ denotes the process time of $O_{i j}$ in the unit that $A M(i, j)$ decides. The key to generate the scheduling scheme is the decoding of operation sequence part.

1. Identify the operation $O_{i j}$ that the first gene $v$ denotes in the operation sequence part of chromosome, remove $v$ from the chromosome and test that weather $O_{i j}$ is the first operation of the task or the unit that belongs, and coupling adjacent predecessor of $O_{i j}$ is not existed.

2. If the condition is satistied, $s_{O_{i j}}=0$, compute the end time of $O_{i j}$; otherwise, compute $e_{J P\left[O_{i j}\right]}, e_{O P\left[O_{i j}\right]}$ and the earliest embeddable interval $\left[p s_{i}, p e_{i}\right]$, $s_{O_{i j}}=\max \left\{e_{J P\left[O_{i j}\right]}, e_{O P\left[O_{i j}\right]}, p s_{i}\right\}$, compute the end time of $O_{i j}$

3. Loop execute the step 1 to 2 , until the operation in operation sequence part of chromosome is empty.

\section{G. Selection Operator Design}

Selection operator simulates natural selection and the performance of chromosome is not a single, which needs to adopt multi-objects technology to design.

\section{1) Fitness Assignment Strategy}

The choice of the scheduling schemes depends on assessment of chromosomes, and Pareto dominate strategy divides chromosomes into different level to sequence the schemes. The paper chooses the NSGA-II method to divide the chromosomes into levels with dominated relationship, and let $Z_{f}$ be the population, $Z_{f}(k)$ be the swarm in non-dominated level $k$.

1. Selects all the non-dominated chromosomes in population $Z_{f}$ to form $Z_{f}(k), k=0$.

2. Selects all the non- dominated chromosomes in swarm $Z_{f}-\bigcup_{i=1}^{k} Z_{f}(k)$ to form $Z_{f}(k)$.

3.Loop execute the step 1 to 2 until $Z_{f}-\bigcup_{i=1}^{k} Z_{f}(k)=\varnothing$. 


\section{2) Diversity Retaining Strategy}

At the basis of non-dominated levels, it still needs to differentiate for the chromosomes in the same level to avoid to converge to a single scheduling scheme and to make the non-dominated chromosomes distributed uniformly in the non-dominated surface, the paper chooses Hamming distance to measure the distances between chromosomes.

The measure of Hamming distance depends on the sum of the same gene with different attribution in chromosomes. Let $x^{p}, x^{q}$ be arbitrary chromosomes in feasible space, $x^{p \text {,allocation }}, x^{q, \text { allocation }}$ denotes operation allocation part, $x^{p \text {,sequence }}, x^{q \text {,sequence }}$ denotes operation sequence part, and the Hamming distance between $X^{p}$ and $x^{q}$ could be described as follow:

$$
\begin{aligned}
& H\left(x^{p}, x^{q}\right)=\sum_{j=1}^{\sum_{i=1}^{n}\left|J_{i}\right|} h\left(x_{j}^{\text {p,allocation }}, x_{j}^{q, \text { allocation }}\right) \\
& +\sum_{j=1}^{\sum_{i=1}^{n}\left|J_{i}\right|} h\left(x_{j}^{p, \text { sequence }}, x_{j}^{q, \text { sequence }}\right)
\end{aligned}
$$

The similarity of $x^{p}$ in non-dominated level $Z_{f}(k)$ is:

$$
S\left(x^{p}\right)=\sum H\left(x^{p}, x^{q}\right), x^{q} \in Z_{f}(k)
$$

The similarity $S\left(x^{p}\right)$ describes the distance between $x^{p}$ and the other chromosomes in swarm $Z_{f}(k)$, which denotes the search degree of direction that $x^{p}$ represented. In order to ensure the uniform distribution of search directions, the paper chooses $S\left(x^{p}\right)$ as the evolution probability of $x^{p}$.

\section{3) Elite Retaining Strategy}

In multi-objects optimization, the contradiction among scheduling objects leads to form numerous non-dominated chromosomes, which could guide search and be benefited to diversity strategy. Therefore, the paper introduces the memory to store the non-dominated chromosomes formed in the search process.

To promote the rate of convergence, memory needs to take part in the selection of chromosomes. Mix $P(T), C(T)$ and memory into mixture population, which would divide into non-dominated swarm $Z_{f}(k)$ based on NSGA-II method.

To avoid to early convergence, $P(T+1)$ needs to be selected respectively in all the swarms. The number of selection in $Z_{f}(k)$ depends on $\left[\left((n-k+1) / \sum_{k=1}^{n} k\right) \times\left|Z_{f}\right|\right]$, where [] denotes the integer part, and the selection probability of chromosome $x^{p}$ in swarm $Z_{f}(k)$ is $S\left(x^{p}\right)$.
After population selection, update the memory according to non-dominated swarm $Z_{f}(1)$.

\section{H. Terminal Condition Design}

The contradiction among scheduling objects makes the terminal condition design could only refer to the swarm search information obtained in search process, the paper chooses number of Iterations hits the set point as the terminal condition.

Comprehensive the design of algorithm execution step, the multi-objects scheduling optimization algorithm flow chart is shown in figure 1 .

\section{CASE ANALYSis}

\section{A. Aircraft Maintenance Shop Scheduling Case Analysis}

To examine the applicability of multi-objects scheduling optimization algorithms, the paper takes aircraft maintenance shop flow for reference to create the formalization model, which is shown in figure 2, the process time of unit and operation is shown as table 1 .

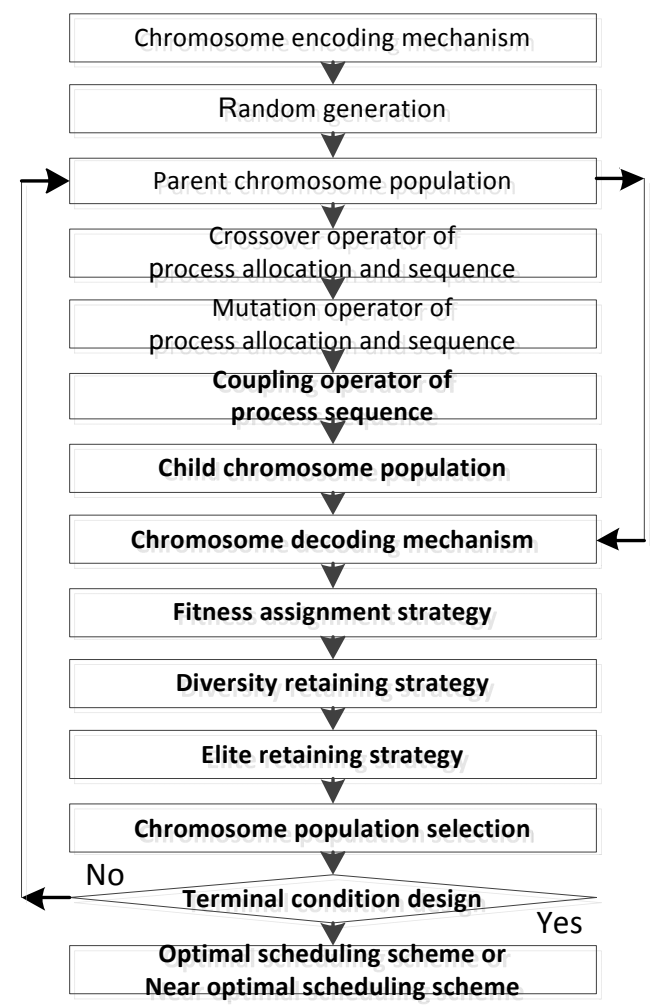

FIGURE I. ALGORITHM FLOWCHART OF MULTI-OBJECTS SCHEDULING OPTIMIZATION ALGORITHM

The paper designs program in java, and adopts the computer with Intel(R) Core(TM)i3-2310M 2.10GHz CPU and 2.00G memory to run the case.

Let the scale of population be $5 \times(n+m)$, crossover probability be $90 \%$, mutation probability be $20 \%$, iteration 
point be 500 , and $f_{1}, f_{2}, f_{3}$ are adapted as scheduling objects. The experimental result is shown in table 2, figure 3.

Compositing the experimental results, scheduling schemes obtained makes clear that multi-objects scheduling optimization algorithm the paper designed is suit to solve aircraft maintenance shop scheduling problem. Compositing the 3-D perspective of distribution of non-dominated chromosomes in state space, the optimal surface of coupling constraints is not present as a smooth surface, which indicates that coupling constraints increases the warp degree of feasible space. In the meantime, the strategy of generating only active schedules in chromosome decoding mechanism will promote efficiency, but will also lead to the situation that different chromosomes representative the same scheduling scheme.

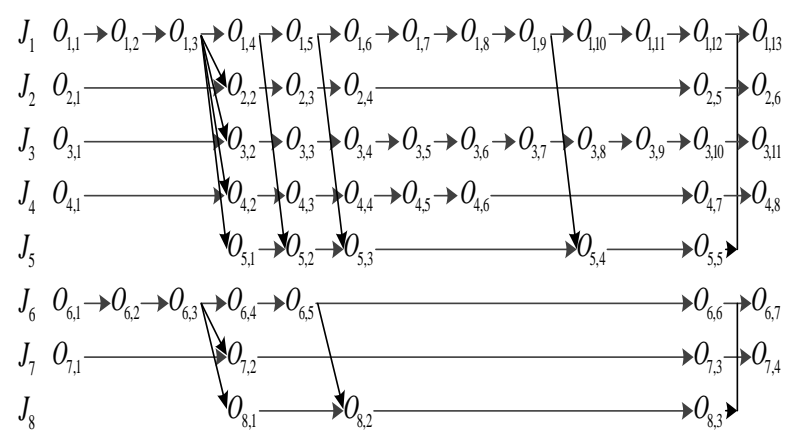

FIGURE II. TASK SET, OPERATION SET AND CHAIN CONSTRAINT, COUPLING CONSTRAINT

TABLE I. PROCESS TIME OF UNIT AND OPERATION

\begin{tabular}{|c|c|c|c|c|c|c|c|c|c|c|c|c|c|c|c|c|c|c|c|}
\hline & $M_{1}$ & $M_{2}$ & $M_{3}$ & $M_{4}$ & $M_{5}$ & $M_{6}$ & & $M_{7}$ & $M_{8}$ & & $M_{9}$ & $M_{10}$ & $M_{11}$ & & $M_{12}$ & $M_{13}$ & & $M_{14}$ & $M_{15}$ \\
\hline$O_{1,1}$ & 30 & 30 & - & - & - & - & $\mathrm{O}_{2,1}$ & 30 & - & $\mathrm{O}_{3,1}$ & 30 & - & - & $O_{4,1}$ & 30 & - & $O_{5,1}$ & 25 & 28 \\
\hline$O_{1,2}$ & 23 & 29 & 14 & 30 & 20 & 22 & $\mathrm{O}_{2,2}$ & 40 & 39 & $\mathrm{O}_{3,2}$ & 14 & 20 & 13 & $O_{4,2}$ & 14 & 13 & $O_{5,2}$ & 32 & 27 \\
\hline$O_{1,3}$ & 60 & 70 & 49 & 62 & 50 & 70 & $\boldsymbol{O}_{2,3}$ & 43 & 49 & $\mathrm{O}_{3,3}$ & - & 11 & 14 & $\mathrm{O}_{4,3}$ & 12 & 15 & $O_{5,3}$ & 25 & 31 \\
\hline$O_{1,4}$ & 35 & 46 & 37 & 25 & - & 28 & $\mathrm{O}_{2,4}$ & 16 & 19 & $\mathrm{O}_{3,4}$ & 20 & 15 & 18 & $\mathrm{O}_{4,4}$ & 36 & 27 & $O_{5,4}$ & 24 & 21 \\
\hline$O_{1,5}$ & 46 & 37 & 42 & 35 & 50 & 25 & $\boldsymbol{O}_{2,5}$ & 26 & 29 & $\mathrm{O}_{3,5}$ & 14 & - & 21 & $O_{4,5}$ & 24 & 21 & $O_{5,5}$ & 12 & 15 \\
\hline$O_{1,6}$ & 55 & 62 & 42 & - & 57 & 56 & $\mathrm{O}_{2,6}$ & 60 & - & $\mathrm{O}_{3,6}$ & 12 & 14 & 11 & $O_{4,6}$ & 15 & 20 & $O_{8,1}$ & 24 & 25 \\
\hline$O_{1,7}$ & 40 & - & 26 & 37 & 31 & 29 & & & & $\mathrm{O}_{3,7}$ & - & 22 & 25 & $\mathrm{O}_{4,7}$ & 23 & 17 & $O_{8,2}$ & 36 & 39 \\
\hline$O_{1,8}$ & - & 35 & 17 & 42 & 32 & 41 & & & & $\mathrm{O}_{3,8}$ & 12 & 11 & - & $O_{4,8}$ & 60 & - & $O_{8,3}$ & 12 & 14 \\
\hline$O_{1,9}$ & 30 & 21 & 34 & 26 & - & 35 & & & & $\mathrm{O}_{3,9}$ & 12 & 14 & 15 & & & & & & \\
\hline$O_{1,10}$ & 52 & 43 & 38 & 48 & 50 & 52 & & & & $\mathrm{O}_{3,10}$ & 37 & 26 & 40 & & & & & & \\
\hline$O_{1,11}$ & 93 & - & 85 & 80 & 94 & 83 & & & & $O_{3,11}$ & 60 & - & - & & & & & & \\
\hline$O_{1,12}$ & 31 & 26 & 22 & 30 & 25 & 27 & & & & $O_{7,1}$ & 24 & 21 & 32 & & & & & & \\
\hline$O_{1,13}$ & 60 & 60 & - & - & - & - & & & & $O_{7,2}$ & 14 & 13 & 15 & & & & & & \\
\hline$O_{6,1}$ & 24 & 35 & 31 & 24 & 19 & 21 & & & & $O_{7,3}$ & 32 & 28 & 25 & & & & & & \\
\hline$O_{6,2}$ & 14 & 13 & 16 & 10 & 12 & 11 & & & & $\mathrm{O}_{7,4}$ & 35 & 21 & 26 & & & & & & \\
\hline$O_{6,3}$ & 21 & 23 & 25 & 20 & 24 & 22 & & & & & & & & & & & & & \\
\hline $\mathrm{O}_{6,4}$ & 14 & 21 & 15 & 23 & 13 & 17 & & & & & & & & & & & & & \\
\hline$O_{6,5}$ & 25 & 21 & 26 & 21 & 24 & 22 & & & & & & & & & & & & & \\
\hline$O_{6,6}$ & 31 & 25 & 36 & 32 & 27 & 25 & & & & & & & & & & & & & \\
\hline$O_{6,7}$ & 37 & 31 & 32 & 36 & 31 & 38 & & & & & & & & & & & & & \\
\hline
\end{tabular}

TABLE II. SCHEDULING SCHEME PERFORMANCE OF COUPLING CONSTRAINT MODEL

\begin{tabular}{cccccc}
\hline$f_{1}$ & $f_{2}$ & $f_{3}$ & $f_{1}$ & $f_{2}$ & $f_{3}$ \\
\hline 449 & 198 & 1494 & 459 & 168 & 1491 \\
480 & 134 & 1524 & 473 & 136 & 1511 \\
480 & 135 & 1523 & 461 & 158 & 1499 \\
466 & 133 & 1529 & 477 & 142 & 1499 \\
480 & 133 & 1526 & 479 & 138 & 1496 \\
466 & 134 & 1527 & 474 & 146 & 1497 \\
466 & 156 & 1525 & 465 & 162 & 1498 \\
466 & 135 & 1526 & 457 & 168 & 1486 \\
466 & 135 & 1526 & 459 & 158 & 1498 \\
449 & 198 & 1501 & 457 & 166 & 1491 \\
475 & 162 & 1497 & 467 & 152 & 1501 \\
& \multicolumn{5}{c}{$\Delta=0.62748$} \\
\end{tabular}
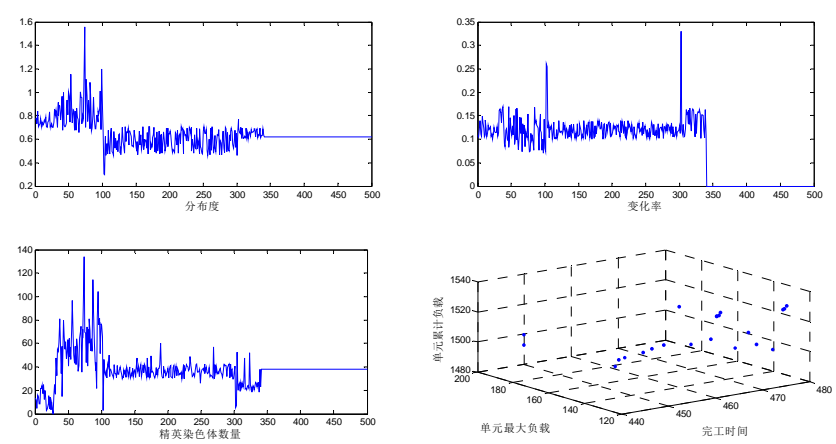

FIGURE III. TEST PROCESS OF COUPLING CONSTRAINT SCHEDULING

\section{SUMMARY}

To make the maintenance adapt for modern aircraft, the modeling and scheduling algorithm of aircraft maintenance shop scheduling problem are discussed. First of all, at the base of maintenance shop scheduling modeling referring to the formalization of flexible job shop scheduling problem, the connection and the distinction between chain constraint and 
coupling constraint are discussed. In the next place, genetic algorithm is adopted as framework, where chromosome encoding and decoding mechanism, scheduling scheme initialization, crossover operator, mutation operator and selection operator are all designed, and the coupling operator is introduced to adapt the operation sequence part of chromosome to avoid the situation that the chromosome might violate the coupling constraint and could not decode. At the end, the maintenance shop scheduling case is established to examine the applicability and quality of multi-objects scheduling optimization algorithms.

\section{REFERENCES}

[1] Srinivas N, Deb K. Multi-objective optimization using non-dominated sorting in genetic algorithms. Evolutionary Computation. 1994, 2(3):221-248.

[2] Zitzler E, Thiele L. Multiobjective evolutionary algorithms: a comparative case study and the strength pareto approach. IEEE Transactions on Evolutionary Computation. 1999, 3(4):257-271.

[3] Zitzler E, Laumanns M, Thiele L. SPEA2: improving the strength pareto evolutionary algorithm. TIK-Report 103. 2001.

[4] KNOWLES J, CORNE D. The Pareto archived evolution strategy: a new baseline algorithm for multi-objective optimization[C]. Proceedings of the 1999 Congress on Evolutionary Computation. Piscataway, NJ: IEEE Press, 1999: 98-105.

[5] Deb K, Pratap A, Agarwal S, Meyarivan T. A fast and elitist multi-objective genetic algorithm NSGA-II. IEEE Transaction on Evolutionary Computation. 2002, 6(2):182-197.

[6] Mark Erickson, Alex Mayer, Jeffrey Horn. The niched pareto genetic algorithm 2 applied to the design of groundwater remediation systems[C]. In: Proceedings of the 1st International Conference on Evolutionary Multi-criterion Optimization. 2001, 681-695. 